\title{
Detección molecular de Candidatus liberibacter solanacearum (Zebra Chip) en papa (Solanum tuberosum L,) en Nicaragua
}

\section{Molecular detection of Candidatus liberibacter solanacearum (Zebra Chip) in patato (Solanum tuberosum $\mathbf{L}$ ) in Nicaragua}

\begin{abstract}
Jenifer Bermania Ramírez Durán, Andrea Zamora Jarquín², Isaías Sánchez Gómez ${ }^{3}$, Jorge Ulises Blandón Díaz ${ }^{4}$
${ }^{1}$ Ing. Sistemas de protección agrícola y forestal

${ }^{2}$ MSc. Fitopatología, Instituto Nicaragüense de Tecnología Agropecuaria, km 14.1 carretera norte, 3 km al sur Managua, Nicaragua, e-mail: andrea.zamora0301@gmail.com

${ }_{3}^{3}$ MSc. Sanidad vegetal, Universidad Nacional Agraria, ORCID: https://orcid.org/0000-0002-6604-1660 / e-mail: isanchez@ci.una.edu.ni.

${ }^{4}$ Ph.D. Fitopatología, Universidad Nacional Agraria, ORCID: https://orcid.org/0000-0002-7904-8853, e-mail: ulisesdb@ci.una.edu.ni.
\end{abstract}
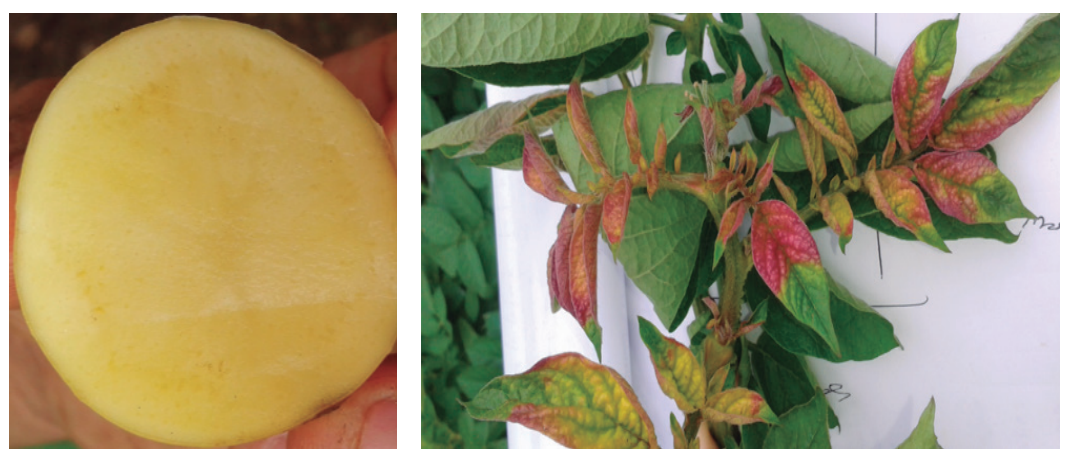

\section{RESUMEN}

En el cultivo de papa se han registrado diversos problemas fitosanitarios importantes por la presencia de virus, bacterias y hongos. En los últimos años, productores de los departamentos de Estelí y Jinotega han reportado plantas con síntomas de la enfermedad Zebra chip ocasionada por la bacteria fastidiosa Candidatus liberibacter solanacearum. La enfermedad Zebra chip ha causado grandes pérdidas económicas a los productores de papa del país donde la producción se desarrolla todo el año. Ante esta problemática el Instituto Nicaragüense de tecnología Agropecuaria (INTA) junto con la Universidad Nacional Agraria (UNA) realizó un estudio comprendido entre los meses de agosto 2015 a Julio 2016 con el objetivo de generar información relacionada a la presencia y distribución de $\mathrm{Ca}$. L. solanacearum en los departamentos mencionados. Se colectaron 162 muestras de tejidos con síntomas de la enfermedad en cuatro localidades de Jinotega y tres localidades de Estelí durante los tres ciclos de siembra del cultivo. Las muestras de tejido fueron procesadas y analizadas en el Centro Nacional de Investigación Agropecuaria y Biotecnología (CNIA-INTA) para detectar el agente causal Ca.l. solanacearum utilizando los cebadores moleculares ligados a la bacteria Lso Tx 16/23 y al gen de limpieza Lso adk. 50 muestras

\begin{abstract}
Several important phytosanitary problems have been recorded in potato cultivation due to the presence of viruses, bacteria and fungi. In recent years, producers in the departments of Estelí and Jinotega have reported plants with symptoms of the Zebra disease chip caused by the fastidiosa bacterium Candidatus liberibacter solanacearum. The Zebra chip disease has caused large economic losses to potato producers in the country where the production is developed all year. Faced with this problem, the Nicaraguan Institute of Agricultural Technology (INTA), together with the National Agrarian University (UNA), conducted a study between August 2015 and July 2016 with the objective of generating information related to the presence and distribution of Ca. Solanacearum in the above mentioned departments. A total of 162 tissue samples with disease symptoms were collected from four locations in Jinotega and three locations in Estelí during the three cropping cycles. Tissue samples were processed and analyzed at the National Center for Agricultural Research and Biotechnology (CNIA-INTA) to detect causative agent $\mathrm{Ca}$.1. Solanacearum using the molecular primers bound to the bacterium Lso Tx 16/23 and the cleansing gene Lso adk. 50 samples were positive with the Lso Tx 16/23 primer indicating high speci-
\end{abstract}

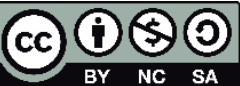

(C) 
resultaron positivas con el cebador Lso Tx 16/23 indicando alta especificidad para la detección de este patógeno, mientras que con los cebadores Lso ADK 42 muestras fueron positivas presentando polimorfismos. Se detectó la bacteria en las zonas de El Tayacán y El Zacatón en el departamento de Estelí y en el departamento de Jinotega El Mojón, La Palmera y San Gregorio. El mayor porcentaje de afectación por Ca. L. solanacearum se presentó en Jinotega con un $52.17 \%$ en comparación con Estelí que presento un $22.41 \%$ de las muestras colectadas.

Palabras clave: Cebador, Lso TX 16/23, Lso ADK, bacteria, agente causal. ficity for the detection of this pathogen, whereas with the Lso ADK primers 42 samples were positive presenting polymorphisms. The bacterium was detected in the areas of El Tayacán and El Zacatón in the department of Estelí and in the department of Jinotega El Mojón, La Palmera and San Gregorio. The highest percentage of affection for Ca. L. solanacearum was presented in Jinotega with $52.17 \%$ compared to Estelí that presented $22.41 \%$ of the samples collected. Key words: Primer, Lso TX 16/23, Lso ADK, bacterium, causal agent.

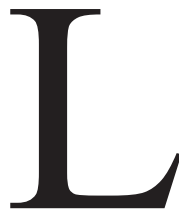

a papa es el cuarto cultivo sembrado en más de cien países, siendo el alimento básico de los países desarrollados (Europa y Estados Unidos), quienes consumen $75 \mathrm{~kg}$ per cápita anuales. La importancia de la papa radica en que sus tubérculos son parte de la dieta de millones de personas a nivel mundial ya que contiene $80 \%$ de agua y la materia seca está constituida por carbohidratos, proteínas, celulosa, minerales, vitaminas A y $\mathrm{C}$ que proporcionan una dieta balanceada (INTA, 2004). La papa se cultiva únicamente en elevaciones que oscilan entre 700 a 1,300 msnm con temperaturas promedio de $18^{\circ} \mathrm{C}$ lo que la hace apta para su buen desarrollo (FUNICA, 2007).

En Nicaragua, la papa representa un cultivo de mucha importancia después de los granos básicos y se cultiva en climas templados de Estelí, Jinotega y Matagalpa. El consumo per cápita en nuestro país es de $8 \mathrm{~kg}$ anuales. Se siembran entre 800-1200 ha con una producción que satisface el $35-40 \%$ de la demanda nacional (INTA, 2004). El país importa de $30-40 \%$ de papa fresca y $100 \%$ de tubérculo-semilla para cubrir la demanda del mercado (FUNICA, 2007). Entre los factores que limitan la producción de papa en Nicaragua se pueden citar la escasez de semilla, el alto costo, la baja calidad de tubérculos por la proliferación de enfermedades y el costo de la aplicación obligatoria de agroquímicos para el control de plagas y enfermedades (INTA, 2004).

Desde el comienzo del año 2011 en las plantaciones de papa de Nicaragua se observó sintomatología de la enfermedad zebra chip causada por la bacteria fastidiosa Ca. I solanacearum y con ella la presencia prolífera del insecto Bactericera cockerelli este insecto al alimentarse de la planta le transmite la bacteria causante de la enfermedad (Toledo, 2011). Para este entonces la enfermedad había sido reportada en otros países tales México (Leyva-Lopez et al., 2002, Martínez-Soriano et al., 1999), Estados Unidos (Banttari et al., 1990, Lee et al., 2009), Canadá (Khadhair et al., 1997; Khadhair \& McAllister,
2003), Bolivia (Jones et al., 2005), Rusia (Girsova et al., 2008), Australia (Harding \& Teakle, 1985), Korea (Jung et al., 2003), y Nueva Zelanda (Liefting et al., 2009). En Nicaragua no hay reportes cuantificables de las pérdidas a causa de la enfermedad Zebra chip que está asociada a la papa, pero las plantaciones han sido gravemente infectadas al punto de reportarse pérdidas totales. Según $\mathrm{Gu}$ tiérrez et al.; (2013) en México la producción de papa ha sufrido pérdidas económicas de $70 \%$ a $90 \%$ causado por esta enfermedad.

Ca. Liberibacter solanacearum es una bacteria que se aloja en los tubos cribosos del floema y afecta a distintos cultivos pertenecientes a la familia de las rutáceas, solanáceas y apiáceae. A través de estudios filogenéticos de la región 16S rRNA se ha encontrado que $C a$. L. solanacearum pertenece a las $\alpha$-proteobacterias y está muy relacionada a 4 especies de Candidatus liberibacter: Ca. L. asiaticus, Ca. L. africanus, Ca. L. americanus y Ca. L. europaeus. Es una bacteria Gram negativa y logra su desarrollo óptimo entre $27-32^{\circ} \mathrm{C}$ (Camacho y Tapia et al., 2016). Esta enfermedad está caracterizada por los síntomas de amarillamiento, clorosis con los bordes de las hojas moradas, achaparramiento de la planta, abultamiento del tallos en lugares de inserción, formación de tubérculos aéreos (Hernández, 2012), también numerosos tubérculos pequeños mal formados y de mala calidad, retraso del crecimiento de la planta, rotación ascendentes de hojas, entrenudos acortados y la proliferación de brotes axilares y en los tubérculos se observa decoloración y un ligero rayado oscuro, y cuando se fríen las rayas son más dominantes afectando también el sabor lo que hace que lo hace comercialmente inaceptable (Munyaneza, 2012).

Las técnicas moleculares para la detección de hongos y virus se han implementado recientemente en $\mathrm{Ni}$ caragua en cultivos de frijol, tomate, cacao y papa. Sin embargo, las bacterias fastidiosas como patógenas no han sido de mucha importancia en cultivos agrícolas. Es importante para los productores y gobierno confirmar si los 
síntomas pertenecen Zebra, por tanto este primer estudio tiene por objetivo detectar la presencia y distribución de Candidatus Liberibacter solanacearum agente causal de la enfermedad Zebra chip en el cultivo de papa, en los departamentos de Jinotega y Estelí, mediante la técnica de reacción en cadena de la polimerasa (PCR, por sus siglas en inglés)

\section{MATERIALES Y MÉTODOS}

Ubicación y periodo del estudio. La investigación se realizó en el (CNIA-INTA), ubicado en el departamento de Managua, $\mathrm{km} 14.1$ carretera norte, $3 \mathrm{~km}$ hacia el sur. Localizado entre la Latitud: $12^{\circ} 08^{\prime} 36^{\prime \prime} \mathrm{N}$ y Longitud: $86^{\circ} 09^{\prime} 49^{\prime \prime} \mathrm{W}$, con una elevación de $56 \mathrm{msnm}$ en el departamento de Managua. Este trabajo se realizó durante los meses de agosto 2015 a julio 2016.

Recolección de muestras. Se colectaron 162 muestras de tejido con síntomas característicos de la enfermedad en cuatro localidades del departamento de Jinotega y tres en el departamento de Estelí en los periodos de primera, postrera y apante. Las variedades de papa presentes en las zonas de muestreo fueron: Granola, Desiree, Calwhite, Silvana, Picaso, Provento, Toluca, Punta roja, Ronaldo, Monte Carlo. Cada uno de los sitios de donde se recolectaron las muestras fue geo-referenciado mediante un dispositivo de Sistema de posicionamiento global (GPS). Se recolectaron muestras que presentaban los síntomas característicos de la enfermedad, los cuales consistían en: amarillamiento, clorosis con los bordes de las hojas moradas, achaparramiento de la planta, abultamiento de los tallos en lugares de inserción, formación de tubérculos aéreos, decoloración en los tubérculos con forma de rayado ligero. Las muestras se colocaron en bolsas de papel y bolsas plásticas con cierre hermético dentro de una hielera para ser trasladadas al laboratorio y fueron almacenadas a $-20^{\circ} \mathrm{C}$ para después proceder a extraer ADN.

Extracción de ADN. Para la extracción de ADN se utilizó el método descrito por Dallaporta et al., (1983) con algunas modificaciones. Por cada genotipo se tomó $5 \mathrm{mg}$ de tejido vegetal y se colocó en un tubo eppendorf (1.5 ml). Se agregó buffer de extracción Tris- HCL $100 \mathrm{mM}$, EDTA $50 \mathrm{mM}, \mathrm{NaCl} 500 \mathrm{mM}$ y 2-mercaptoetanol $20 \mathrm{mM}$ a cada muestra y luego se maceró el tejido usando pistilos plásticos estériles hasta homogenizar las muestras. Seguidamente, se agregó sulfato dodecil sódico (SDS) al 10\% y se colocaron las muestras en incubadora tipo baño María a $65^{\circ} \mathrm{C}$ por 10 minutos agitándola por lo menos tres veces. Se adicionó acetato de potasio $(3 \mathrm{M})$ a cada muestra para incubarse a menos $20^{\circ} \mathrm{C}$ por 20 minutos, posteriormente las muestras se centrifugaron a $15,000 \mathrm{rpm}$ por $15 \mathrm{minu}-$ tos a $5^{\circ} \mathrm{C}$. Se extrajo el sobrenadante y se transfirió a un tubo eppendorf estéril y se procedió a agregar y mezclar 0.6 volúmenes de 2-propanol helado, se conservaron las muestras a menos $20^{\circ} \mathrm{C}$ por 20 minutos y las muestras se volvieron a centrifugar a $15,000 \mathrm{rpm}$ por 15 minutos a $5^{\circ} \mathrm{C}$. Se procedió a extraer el 2-propanol volteando el tubo rápidamente, luego se lavó dos veces el pellet en etanol al $70 \%$ a $15,000 \mathrm{rpm}$ a $5^{\circ} \mathrm{C}$ por 5 minutos, se secaron los pellets de ADN a temperatura ambiente por 50 minutos. Los pellets de ADN se re disolvieron en buffer TE $1 \mathrm{X}$ (conteniendo Tris- HCL $(100 \mathrm{mM}) \mathrm{pH} 8.0$ y EDTA $(1 \mathrm{mM})$ y se conservaron las muestras a menos $20^{\circ} \mathrm{C}$ para su posterior utilización.

Identificación molecular de Candidatus liberibacter solanacearum. La amplificación PCR realizada con los cebadores Lso adk fue a un volumen final de $20 \mu 1$ utilizando $5 \mu \mathrm{M}$ del cebador delantero y reverso (Lso adk F 5'-GCGCCACACTAACATCTCCTTCC-3'y Lso adk R 5'-CGCAGCAGTATGAGGGCC-3'), $1 \mathrm{X}$ de buffer, 200 $\mu \mathrm{M}$ de cada dNTPs $(10 \mathrm{Mm})$ y $0.02 \mathrm{U} / \mu 1$ de Taq polimerasa, agua libre de nucleasas y ADN. El desarrollo de la PCR se realizó en un termociclador Eppendorf bajo las siguientes condiciones: desnaturalización inicial 30 segundos a $98^{\circ} \mathrm{C}$, seguido de 40 ciclos a $98^{\circ} \mathrm{C}$ por 10 segundos, $60^{\circ} \mathrm{C}$ por 20 segundos, $72^{\circ} \mathrm{C}$ por 30 segundos y una extensión final a $72^{\circ} \mathrm{C}$ por 7 minutos.

En el caso de la amplificación PCR realizada con los cebadores Lso TX 16/23 fue a un volumen final de 20 $\mu 1$ utilizando 10 pmol del cebador delantero y reverso (Lso TX 16/23 F (5' - AATTTTAGCAAGTTCTAAGGG-3' y R Lso TX 16/23 5'-GGTACCTCCCATACGC-3'), Go taq colorless master mix 2X, agua libre de nucleasas y ADN. El desarrollo de la PCR se realizó en un termociclador Eppendorf bajo las siguientes condiciones: desnaturalización inicial 30 segundos a $98^{\circ} \mathrm{C}$, seguido de 40 ciclos a $98^{\circ} \mathrm{C}$ por 10 segundos. $55^{\circ} \mathrm{C}$ por 20 segundos, $72^{\circ} \mathrm{C}$ por 30 segundos y una extensión final a $72{ }^{\circ} \mathrm{C}$ por 7 minutos (Ravindran et al., 2011)

En ambos productos PCR, se visualizaron los resultados colocando 20 ul de las muestras con 4 ul de $6 \mathrm{X}$ loading Dye en un gel de agarosa al $1.2 \%$ que fue teñida con bromuro de etidium antes de solidificar, el gel se colocó en una cámara de electroforesis con buffer TBE $0.5 \mathrm{X}$ se corrió durante 90 min a 90 voltios, y se colocó en un transluminador para verificar la presencia o ausencia de la bacteria en las muestras y se registró mediante fotografía. Se consideraron muestras positivas a las que tuvieron una 
amplificación de fragmentos de acuerdo a los cebadores utilizados Lso TX 16/23 que amplifica a 383 pb y Lso adk que amplifica a $770 \mathrm{pb}$.

\section{Distribución geográfica de Candidatus liberibacter so-} lanacearum. La determinación de la distribución geográfica, se geo referencio mediante un dispositivo de sistema de posicionamiento global (GPS). Tomando los puntos en las plantas con síntomas característicos de la enfermedad.

\section{RESULTADOS Y DISCUSIÓN}

Presencia de Candidatus liberibacter solanacearum con los cebadores Lso TX 16/23. Se procesaron 162 muestras de tejido con síntomas de la enfermedad punta morada y mediante la PCR se detectaron 50 muestras infectadas con Ca Liberibacter solanacearum con el cebador Lso Tx $16 / 23$, representando el $30.88 \%$ de muestras colectadas en los departamentos de Estelí y Jinotega en los tres ciclos del cultivo. Los mayores porcentaje de muestras positivas se observaron en postrera $(9.25 \%)$ en el departamento de Estelí y $8.02 \%$ en primera en el departamento de Jinotega. El cebador Lso TX 16/23 amplificó un fragmento de $383 \mathrm{pb}$ el correspondiente para detectar $\mathrm{Ca}$. Liberibacte solanacearum (figura 1). Los resultados fueron similares a los obtenidos por Ravindran et al., (2011), obteniendo amplificados de $383 \mathrm{pb}$ de la región $16 \mathrm{~S}$ y $23 \mathrm{~S}$ rDNA para Ca. L. solanacearum en el cultivo de la papa. Por otro lado, se han realizado otros estudios de detección de Ca. L. solanacearum en los cultivos de apio y perejil utilizando el marcador Lso TX 16/23 obteniéndose como resultado una alta sensibilidad y especificidad en detección, superando a los marcadores OA2 y OI2c, que son utilizados comúnmente para la detección y diagnóstico de Ca. L. solanacearum (Teresani et al., 2014; Monger y Jeffries, 2016).
Un segundo marcador fue utilizado para la detección de ' $C a$. L. solanacearum. El marcador Lso adk proviene del gen de limpieza adk (housekeeping gene adk) de secuencias alineadas de Ca. L. solanacearum y asiaticus (Ravindran et al., 2011). Un total de 72 muestras de tejido infectado con zebra chip fue utilizado para la detección con el marcador Lso adk obteniendo como resultado 42 muestras positivas, observándose fragmentos de 383, 500 y $770 \mathrm{pb}$. (figura 2). Los resultados se compararon con los obtenidos por Ravindran et al., (2011) encontrando similitud en 9 muestras con una sola banda de $770 \mathrm{pb}$. Sin embargo, la presencia de fragmentos polimórficos con el marcador Lso adk no fue reportado en el estudio de Ravindra et al., 2011 y 2012. De acuerdo a Glynn et al., 2012; los genes de limpieza son generalmente utilizados como tipo de secuencia multilocus (MLST) debido a que experimentan variaciones relativas en la secuencia entre los loci lo cual refleja adaptación de los patógenos y son informativos para identificar grupos entre una población dada.

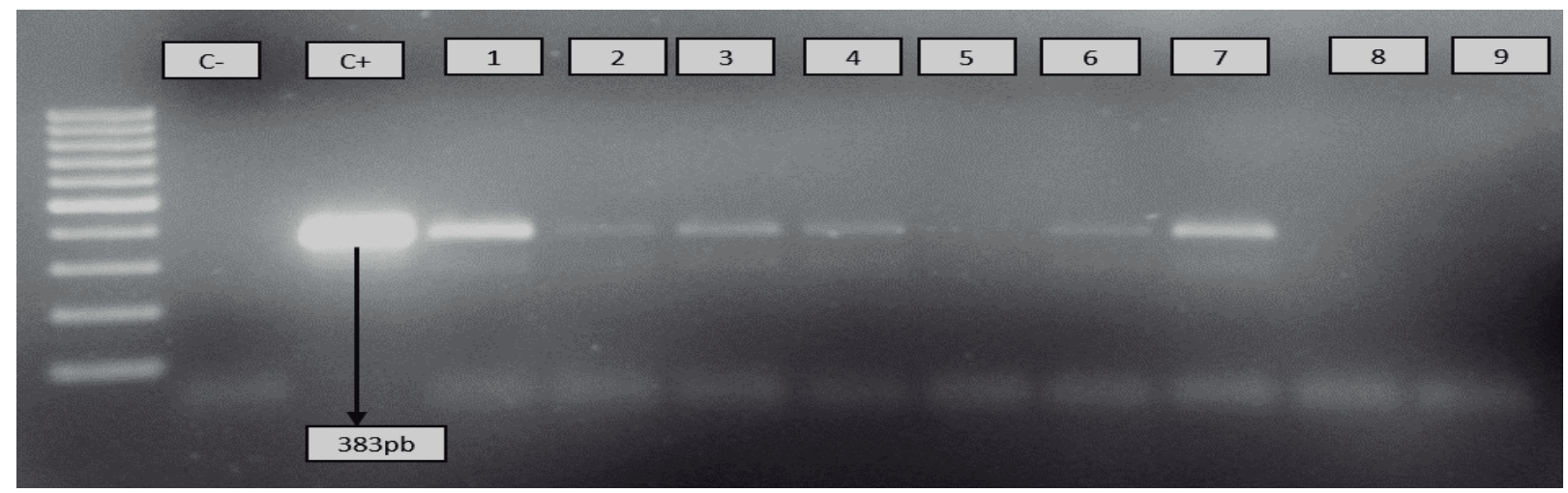

Figura 1. Resultados positivos con cebadores Lso Tx 16/23 (383pb) en muestras de tejido con síntomas de enfermedad (1-9). Control positivo $(\mathrm{C}+)$ y control negativo (C-). 
Distribución geográfica de Candidatus liberibacter solanacearum (CLs). La distribución geográfica se basa fundamentalmente en los informes recolectados de la presencia de Bactericera cockerelli quien transmite la bacteria Candidatus liberibacter solanacearum (MAG Costa Rica, 2010). Mediante los resultados obtenidos de la PCR, que se realizó en muestras colectadas con síntomas de la enfermedad en los departamentos de Jinotega y Estelí se detectó la presencia de Candidatus liberibacter solanacarum en El Tayacán y El Zacatón en el departamento de Estelí, mientras en que el departamento de Jinotega en las zonas del Mojón, La Palmera y San Gregorio.

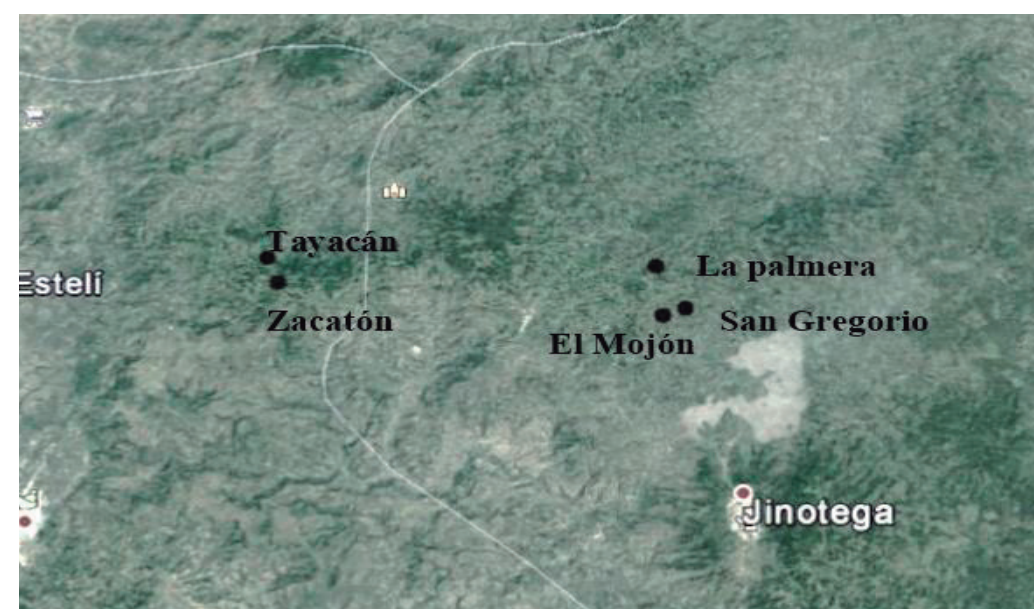

Figura 3. Localidades de los departamentos de Estelí y Jinotega con resultados positivos mediante la técnica PCR para Candidatus liberibacter solanacearum (Puntos negros en el mapa).

Los síntomas de la enfermedad se registraron por primera vez en los campos comerciales de Texas, México en 1994 y ha sido reportada en California, Colorado, Kansas, Nebraska, Nevada y Nuevo México suroeste de Estados Unidos en el 2000, también se registró la presencia en Guatemala, Honduras y Nicaragua en el 2010; en el 2008 se encontró que la bacteria estaba afectado los cultivos en nueva Zelanda, Finlandia, Noruega y Suecia (Secor et al 2009; Munyaneza et al., 2012).

La presencia de Candidatus liberibacter solanacearum en los campos de papa esta correlacionados con la presencia del insecto vector Bactericera cockerelli el cual tiene un rango óptimo de temperatura $21-27^{\circ} \mathrm{C}$, mientras que las temperatura superiores a $32^{\circ} \mathrm{C}$ perjudica la ovoposición y eclosión de $B$. cockerelli (OIRSA, 2015). La altura promedio en el departamento de Estelí es de 900-1500 msnm, mientras que en Jinotega altura promedio oscila entre 700-1700 msnm, según Rubio et al., (2011) la población de B. cockerelli disminuye con la altura, por tanto a alturas mayores de 3200 msnm no se presentan daños significativos económicamente.

\section{CONCLUSIONES}

Se confirmó la presencia de $C a$. l. solanacearum en los campos de papa en los departamentos de Estelí y Jinotega, resultando ser más específico el marcador Lso TX 16/23 F/R para detectar ' $C a$. l. solanacearum. Las zonas con presencia de la bacteria fueron El Tayacán y El Zacatón en el departamento de Estelí y en el departamento de Jinotega en las zonas del Mojón, La Palmera y San Gregorio. Se sugiere realizar estudios adicionales de $\mathrm{Ca}$ l. solanacearum en cultivos que no están relacionados con las solanáceas como pe ejil, zanahoria, apio y otras hortalizas de altura debido a que se ha reportado la presencia de este patógeno en otros países infectando estos cultivos.

\section{AGRADECIMIENTO}

Se agradece el apoyo financiero de las autoridades CNIA-INTA para la realización de este estudio. 


\section{REFERENCIAS BIBLIOGRÁFICAS}

Banttari, EE., Orr, PH; Preston, DA. 1990. Purple top as acause of potato chip discoloration. Trans ASAE 33:221-226.

Camacho Tapia, M; Rojas Martínez, RI; Zavaleta Mejía, E; Rebollar Alviter, A; Aranda Ocampo, S; Suárez Espinosa, J. 2016. Biological, ecological, epidemiological and management aspects of Candidatus Liberibacter. Revista Chapingo serie horticultura. 12. (1). 5-16p.

Dallaporta, SJ; Wood, J; Hicks, JB. 1983. A plant DNA minipreparation: version II. Plant Molecular Reporter 1:19-21

FUNICA (Fundación para el Desarrollo Tecnológico Agropecuario y Forestal de Nicaragua). 2007. Análisis de lacadena sub-sectorial de la papa para consumo. Boletín FUNICA. FUNICA, Managua, Nicaragua 48 p.

Girsova, N, Bottner, KD, Mozhaeva, KA; Kastalyeva, TB; Owens, RA; Lee, M. 2008. Molecular detection and identification of group 16SrI and 16SrXII phytoplasmas associated with diseased potatoes in Russia. Plant Dis. 92:654.

Glynn, J; Islam, M; Bai, Y; Lan, S; Wen, A, Gudmestad, N; Civerolo, E; Lin, H. 2012. Multilocus sequence typing of 'Candidatus liberibacter solanacearum' isolates from North America and New Zealand. J. Plant Pathol. 94:223-228.

Gutierrez, IAT; Sánchez, PJR; Laguna, CA; Ramírez, DJF; Balbuena, MA; Alvarado, GOG. 2013. Detección de Ca liberibacter solanacerum y fitiplasma en cultivo de papa (Solanum tuberosum L.) en el valle de Toluca. Colomb. Biotecnol. 15(1):145-149.

Harding, RM; Teakle, DS. 1985. Mycoplasma-like organisms as causal agents of potato purple top in Queensland. Aust. J. Agric. Res. 36:443-449.

Hernández, LS. 2012. Caracterización de las variedades de papa (Solanum tuberosum L.) Utilizadas en el cofre de perote, Veracruz bajo régimen de temporal. Xalapa, Enríquez Veracruz. Universidad Veracruzana Facultad de Ciencias Agrícolas. 85p.

INTA/NORAD Zona B-3. 2004. Programa MIP. Plegables sobre: como muestrear las plagas de nuestros cultivos.

Jung, HY; Hahm, YI; Lee, JT. Hibi, T; Namba, S. 2003. Characterization of a phytoplasma associated with witches'- broom disease of potatoes in Korea. J. Gen. Plant Pathol. 69:87-89.

Khadhair, AH; McAllister, PD. 2003. Transmission and characterization of phytoplasma diseases associated with infected potato cultivars in Alberta. Acta Hortic. 169:167-176.

Khadhair, AH; Hiruki, C; Hwang, SF; Wang, K. 1997. Molecular identification and relatedness of potato witches'-broom phytoplasma isolates from four potato cultivars. Microbiol. Res. 152:281-286.

Lee, IM; Bottner, KD; Sun, M. 2009. An emerging potato purple top disease associated with a new 16SrIII group phytoplasma in Montana. Plant Dis. 93:970

Leyva-López, NE; Ochoa-Sánchez, JC; Leal-Klevezas, DS; Martínez-Soriano, JP. 2002. Multiple phytoplasmas associated with potato diseases in Mexico. Can. J. Microbiol. 48:1062-1068.

Liefting, LW; Veerakone, S; Ward, LI; Clover G, RG. 2009. First report of Candidatus Phytoplasma australiense' in potato. Plant Dis. 93:969

MAG Costa Rica. 2010. SFE desarrolla Plan de Acción ante la cercanía de la Paratrioza (Bactericera cockerelli Sulc.) Costa Rica. No 45. $4 \mathrm{p}$.

Martínez-Soriano, JP; Leyva-López, NE; Zavala-Soto, ME; Berès, M; Leal-Klevezas, DS. 1999. Detección molecular del agente causal del síndrome "bola de hilo" de la papa en semillas infectadas y asintomáticas. Biotecnol. Apl. 16:93-96.

Monger, WA; Jeffries, CJ. 2016. First report of 'Candidatus Liberibacter solanacearum' in parsley (Petroselinum crispum) seed. New Disease Reports. 34. 31.

Munyaneza Joseph, E. 2012. Zebra Chip Disease of potato Biology, Epidemiology, and Management. Publications from USDA AR.

OIRSA (Organismo Internacional Regional de Sanidad Agropecuaria). 2015. El psílido de la papa y tomate bactericera (=Paratrioza) cockerelli (Sulc) (Hemiptera: Triozidae): ciclo biológico; la relación con las enfermedades de las plantas y la estrategia del manejo integrado de plagas en la región del OIRSA. Mexico D.F. Ed. Tauro S.A.

Ravindran, A; Levy, J; Pierson, E; Gross, CD. 2011. Development of primers for improved PCR detection of the potato zebra chip pathogen, 'Candidatus Liberibacter solanacearum'. Plant Disease. 95. (12). 1542-1546p.

Ravindran, A; Levy, J; Pierson, E; Gross, DC. 2012. Development of a loop-mediated isothermal amplification procedure as a sensitive and rapid method for detection of 'Candidatus Liberibacter solanacearum' in potatoes and psyllids. Phytopathology. 102. (9). 899$907 \mathrm{p}$.

Rubio, COA; Almeyda, LIH; Cadena, HMA; Lobato, SR. 2011. Relacion entre Bactericera cockerelli y la presencia de Candidatus psyllaurous en lotes comerciales de papa. Revista México ciencia agrícola. 2. (1).

Secor, GA; Rivera, VV; Abad, JA; Lee, I-M; Clover, GRG; Liefting, LW; Li, X; De Boer, SH. 2009. Association of 'Candidatus Liberibacter solanacearum' with zebra chip disease of potato established by graft and psyllid transmission, electron microscopy, and PCR. Plant Disease. 93. (6). 575-583p.

Teresani, GR; Bertolini, E; Alfaro-Fernández, A; Martínez, C; Tanaka, FAO; Kitajima, EW; Roselló, M; Sanjuán, S; Ferrándiz, JC; López, MM; Cambra, M; Font, MI. 2014. Association of 'Candidatus Liberibacter solanacearum' with a vegetative disorder of celery in Spain and development of a real-time PCR method for its detection. Phytopathology. 104. (8). 804-811 p.

Toledo, M. 2011. Cultivo de papa: Honduras secretaria de agricultura y ganadería dirección de ciencia y tecnología agropecuaria la esperanza (Tesis de grado) intibuca Honduras 78p. 